\title{
Evaluation of superoxide anion level and membrane permeability in the functionally different organs of Triticum aestivum L. exposed to high temperature and antimycin $\mathrm{A}$
}

\author{
A. Batjuka* and N. Škute \\ Laboratory of Molecular Biology and Genetics, Department of Ecology, Institute of Life Sciences and Technology, Daugavpils University, \\ Parades Str.1a, Daugavpils, Latvia, LV-5401
}

The impact of antimycin $A(A A)$ as an activator of the alternative respiratory pathway (AP) on the rate of superoxide anion $\left(\mathrm{O}_{2}^{\circ-}\right)$ generation and membrane permeability was studied in the functionally different organs of wheat seedlings (Triticum aestivum L.) following short-term and long-term high temperature (HT). The results indicated a significant increase in the $\mathrm{O}_{2}^{\circ-}$ generating rate in etiolated wheat seedlings and seedlings grown under normal daylight regime following short-term $(8 \%-37 \%)$ and long-term $(13 \%-$ $65 \%)$ HT depending on the stage of development. The damages induced by HT were related to the permeability of membranes in the functionally different organs of wheat seedlings. However, our results demonstrated that increase in the $\mathrm{O}_{2}^{\circ-}$ generating rate in etiolated wheat seedlings was significantly lower following the combined effect of $\mathrm{AA}$ and HT, than under stressful conditions without AA. Taken together, our observations show that the induction of AP, provoked by AA, prevents over-reduction of the mitochondrial electron transport chain, alleviating reactive oxygen species formation and protecting the etiolated wheat seedlings against oxidative damage.

Keywords: Antimycin A, high temperature, membrane permeability, superoxide anion, Triticum aestivum L.

PLANTS are continuously exposed to detrimental environmental factors which adversely affect their growth and development as well as pose a major threat to agricultural productivity worldwide. Heat stress (HS) represents a widespread global problem that has a generally negative impact on plant growth and limits the propagation of economically important plant species ${ }^{1}$. This environmental stressor leads to direct injuries such as denaturation of proteins, aggregation, increase in the fluidity of membrane phospholipids, and also to indirect damages like

*For correspondence. (e-mail: anna.batjuka@du.lv) enzymes inactivation, inhibition of mitochondrial protein synthesis and loss of plasma membrane integrity ${ }^{2}$. A significant deviation of atmospheric temperature from the optimum may cause irreversible damages of cell redox homeostasis and eventually lead to the destruction of certain cells, tissues and whole plant organisms ${ }^{3}$.

Mitochondria play a significant role in the energy metabolism of plants cells and are involved in the signalling of gene regulation and maintenance of plant resistance to different environmental stressors ${ }^{4}$. Plant responses are linked to a restructuring of metabolic processes, the final stage of which is protection against various environmental stress conditions. There are different mitochondrial energy-dissipation systems, e.g. non-protonpumping $\mathrm{NAD}(\mathrm{P}) \mathrm{H}$ dehydrogenases (npNAD $(\mathrm{P}) \mathrm{H}-\mathrm{DHases})$, alternative electron transfer pathway, uncoupling proteins (UCPs), ATP-sensitive potassium channel $\left(\mathrm{K}_{\mathrm{ATP}}^{+}\right)$, free fatty acids (FFAs) and ADP/ATP-antiporter, which dissipate the free energy released during electron flows into heat, and maintain the energetic and metabolic balance under stress conditions ${ }^{5}$. The possible role of mitochondrial alternative pathway (AP) in the generation of reactive oxygen species (ROS) raises particular interest and has received much attention because its activity may provide stable functioning of the mitochondrial electron transport chain (mETC), alleviating ROS production in the respiratory chain when the ubiquinone (UQ) pool is over-reduced and reducing the development of oxidative stress at the plant cellular and whole tissue level.

Antimycin A (AA) is a fairly potent mETC inhibitor of the bc1 complex which disrupts the electron flow from cytochrome $b$ to cytochrome $c_{1}$ in complex III (cytochrome $c$ oxidoreductase $)^{6}$, and is also used as an inhibitor of cyclic electron flow around PSI (CEF) which inhibits electron flow from recombinant proton gradient regulation (PGR1) to plastoquinone (PQ) and non-photochemical quenching (NPQ) in chloroplasts ${ }^{7}$. It is known that AP in plants can be strongly induced by application of AA that 
increases the level of alternative oxidase (AOX) transcript and protein ${ }^{8-10}$. Our recent study has demonstrated that AP induced by AA contributes to the stabilization of functional status of the photosynthetic apparatus in Triticum aestivum $\mathrm{L}$. under $\mathrm{HS}^{11}$.

The aim of the present study was to evaluate the impact of AA as a possible inductor of a non-phosphorylating AP on the dynamics of superoxide anion $\left(\mathrm{O}_{2}^{\circ-}\right)$ generating rate and cellular membrane permeability in the functionally different organs of $T$. aestivum L. at various stages (from 4 to 12 days) of development following short-term and long-term high temperature (HT).

\section{Materials and methods}

\section{Plant materials, growing conditions and treatments}

In this study, the first leaves and coleoptiles were used as model systems of developing and senescent organs of wheat seedlings (T. aestivum L. cv. Harmony). The grains were sprouted for $24 \mathrm{~h}$ in plastic pots $(19 \times 12 \mathrm{~cm})$ containing wet filter paper in a growth chamber (Sanyo, Japan) at $25^{\circ} \pm 1^{\circ} \mathrm{C}$. After germination, the uniformly germinated wheat seedlings were transferred in plastic pots of diameter $8.5-\mathrm{cm}$ supplemented with freshly prepared solution of AA ( $1 \mathrm{mg} \mathrm{l}^{-1}$; Sigma, USA, ) and in deionized water (control medium), and the growth of seedlings was monitored in a climate chamber $(16 / 8 \mathrm{~h}$ light/dark cycle, temperature $25^{\circ}-26^{\circ} \mathrm{C}$, growth irradiance of $150 \mu \mathrm{mol}$ (photon) $\mathrm{m}^{-2} \mathrm{~s}^{-1}$ and relative humidity $(\mathrm{RH})$ around $75 \%$ ). Etiolated seedlings were grown in darkness at $26^{\circ} \mathrm{C}$. The seedlings were subjected to short-term $(1 \mathrm{~h})$ and prolonged $(24 \mathrm{~h}) \mathrm{HT}\left(42^{\circ} \mathrm{C}\right)$ on the fourth day of development in the presence of AA (1 $\left.\mathrm{mg} \mathrm{l}^{-1}\right)$ and without AA.

\section{Determination of $\mathrm{O}_{2}^{\circ-}$ producing rate}

The $\mathrm{O}_{2}^{\bullet-}$ generating rate in the first leaves and coleoptiles of wheat seedlings was determined by $0.05 \%$ nitroblue tetrazolium (NBT) staining, with some modifications ${ }^{12}$. Fresh plant material (first leaves and coleoptiles) was incubated with and without $1 \mu \mathrm{l} / \mathrm{ml}$ SOD for $1 \mathrm{~h}$ at $25^{\circ} \mathrm{C}$ in incubation buffer $(4 \mathrm{ml})$ with NBT solution. The incubation buffer contained $10 \mu \mathrm{l}$ EDTA, $10 \mathrm{mM} \mathrm{K}_{2} \mathrm{HPO}_{4}$, $1 \mathrm{mg} / \mathrm{ml}$ Triton X-100 and $0.05 \%$ NBT. The rate of $\mathrm{O}_{2}^{--}$ production was determined by the NBT reduction assay. After $1 \mathrm{~h}$ incubation, NBT reduction was monitored spectrophotometrically (UV/VIS Varian Cary 50 Scan, USA) at a wavelength of $530 \mathrm{~nm}$.

\section{Determination of electrolyte leakage}

Permeability of the cell membranes was measured by detecting electrolyte leakage $(E L)^{13}$. Fresh first leaves and coleoptiles were rinsed with deionized water and then immersed in $15 \mathrm{ml}$ of double-deionized water in a test tube and kept at room temperature $\left(25^{\circ} \mathrm{C}\right)$ for $24 \mathrm{~h}$ in darkness (to prevent electrolytes loss induced by the light). After $24 \mathrm{~h}$, the conductivity of the solution (initial conductivity) was measured using an electrical conductivity meter (Mettler Toledo 980-K19/120 Conductivity Cell, Switzerland). Then the tubes were heated at $100^{\circ} \mathrm{C}$ for $15 \mathrm{~min}$ and cooled down to room temperature, and conductivity was remeasured (final conductivity). EL was calculated according to the equation

$$
\operatorname{EL}(\%)=\left(C_{i}-C_{0}\right) /\left(C_{t}-C_{0}\right) \times 100,
$$

where $C_{i}$ is the initial conductivity of the solution, $C_{t}$ the final conductivity after boiling and $C_{0}$ is the conductivity of deionized water.

\section{Statistical analyses}

All data were statistically analysed and presented as mean and standard error (SE) of three replicates. Statistical analysis of variance of the independent data of three replicates was performed using the program Statistica 2010, and significance between treatment means was tested at the $P<0.05$ level of probability.

\section{Results and discussion}

\section{Changes in $\mathrm{O}_{2}^{\circ-}$ producing rate}

It is well documented that mitochondria are a major source of ROS generation in the etiolated seedlings of plants under exposure to HT and low temperature ${ }^{14,15}$. Our results demonstrated that the $\mathrm{O}_{2}^{--}$producing rate increased on an average by $16 \%$ and $37 \%$ respectively, following short-term and prolonged HT in the etiolated developing organs (Figure $1 a$ and $b$ ), indicating oxidative stress. It is likely that the enhanced production of ROS in seedlings under HT is due to disruption of redox homeostasis $^{16}$, disturbances of the mitochondrial metabolism ${ }^{17}$, and an imbalance in antioxidant systems ${ }^{18}$. Other researchers also found that HT elevated ROS generation in winter wheat cultured cells ${ }^{19}$. The most prominent differences in the etiolated developing organs under exposure to short-term HT appeared at the late stages of development; namely the $\mathrm{O}_{2}^{\circ-}$ producing rate increased by $19 \%$ on the eighth and ninth day of development (Figure $1 a$ ). Previous studies also have reported increased $\mathrm{O}_{2}^{\bullet-}$ production in the first leaves of etiolated wheat seedlings at the late stage of development (seventh day) under exposure to $\mathrm{HT}^{20}$. However, Figure $1 c$ shows that the rate of $\mathrm{O}_{2}^{--}$generation increases by $21 \%$ following short-term HT in the developing organs grown under normal daylight. This increase was more pronounced on the fifth and 


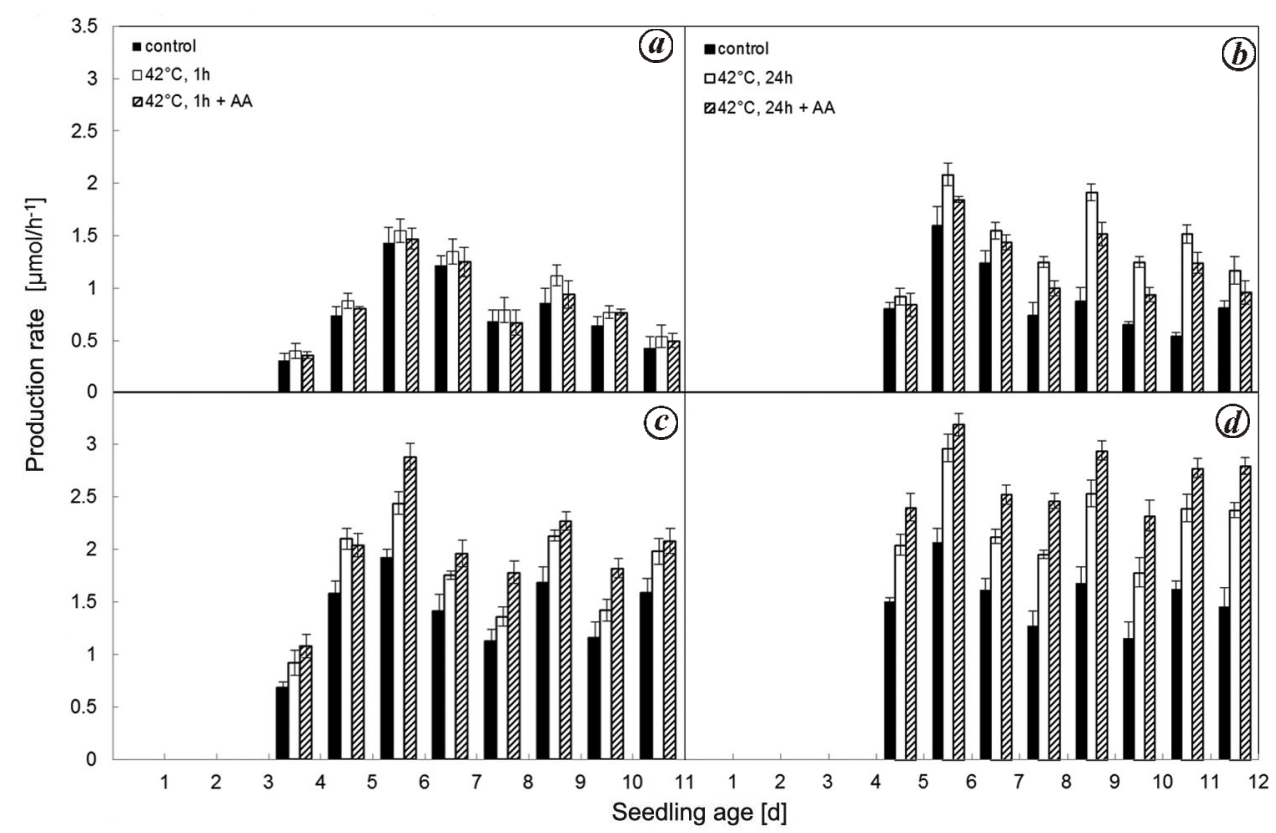

Figure 1. Effect of antimycin A (AA) on superoxide anion $\left(\mathrm{O}_{2}^{--}\right)$production rate in the etiolated developing organs grown under (a) short-term and (b) long-term exposure to high temperature, and under (c) short-term and (d) long-term exposure to high temperature under normal daylight conditions. Data are presented as mean $\pm \operatorname{SE}(n=3)$.

sixth days of development. Moreover, our results indicate that the rate of $\mathrm{O}_{2}^{\circ-}$ generation increases significantly (34\%) following prolonged exposure to elevated temperature in the developing organs grown under normal daylight compared with unstressed seedlings (Figure $1 d$ ), suggesting that serious oxidative injuries and unrecoverable membrane damages have occurred. Presumably, a rapid accumulation of intracellular ROS in the developing organs following HT at the late stages of development plays an essential role in the regulation of senescence and activates programmed cell death (PCD). It is well known that ROS may interact with unsaturated lipids, proteins and nucleic acids (DNA) causing damage to cellular organelles, thus leading to $\mathrm{PCD}^{21}$. Overall, our results assume that PCD in the first leaves of $T$. aestivum $\mathrm{L}$. grown under daylight conditions begins earlier than that in etiolated seedlings, but HT amplifies this process. Evidently, a cascade of enzymatic reactions catalysing the effective inactivation of $\mathrm{O}_{2}^{--}$and other ROS neutralizing systems functions more effectively in the etiolated seedlings of T. aestivum $\mathrm{L}$.

In contrast to the developing organs of wheat seedlings which are relatively long-lived with active cell division, coleoptiles are particularly short-lived organs that senescence rapidly and are degraded during the earliest stages of seedlings development ${ }^{22}$. The results of this study show that the rate of $\mathrm{O}_{2}^{--}$generation does not increase in the etiolated senescent organs following short-term HT at the early stages of development (Figure $2 a$ ). Moreover, the rate of $\mathrm{O}_{2}^{\circ-}$ generation is lower $(16 \%)$ than the control level in the etiolated senescent organs following prolonged exposure to HT at the early stages of development
(Figure $2 b$ ). We suggest that enzymatic antioxidant systems in the senescent organs of wheat plants function more effectively under stress conditions to protect the developing organs from injuries. This coincides with the results of another study, where $\mathrm{O}_{2}^{\bullet-}$ production slightly decreased at the early stages of development in coleoptiles of wheat seedlings under exposure to long-term $\mathrm{HT}^{20}$. However, it is evident from the present study that the rate of $\mathrm{O}_{2}^{\circ-}$ generation significantly increases by $19 \%$ (Figure $2 a$ ) and 29\% (Figure $2 b$ ) respectively, in the etiolated senescent organs at the late stages of development (seventh day) under short-term and long-term exposure to HT in comparison to control. This finding strongly suggests that etiolated senescent organs are more resistant to HT at the early stages of development than at the late stages of ontogenesis. It is well known that chloroplasts produce ROS during cellular stress and the major site of $\mathrm{O}_{2}^{--}$generation is the thylakoid membranebound electron primary acceptor of photosystem I (ref. 23). The results of this study reveal that the rate of $\mathrm{O}_{2}^{\circ-}$ generation increases significantly on average by $25 \%$ and $37 \%$ respectively, following short-term and long-term HT in the senescent organs grown under normal daylight (Figure $2 c$ and $d$ ). Moreover, $\mathrm{O}_{2}^{--}$producing rate at the late stages was more intense than at the early stages of development in the senescent organs following long-term HT. An increase in the rate of $\mathrm{O}_{2}^{--}$generation in the senescent organs of wheat seedlings can be linked with the dysfunction of chloroplasts and acceleration of senescence under exposure to HT. We envisage that PCD in the senescent organs like in the developing organs of wheat seedlings under normal daylight begins earlier than 


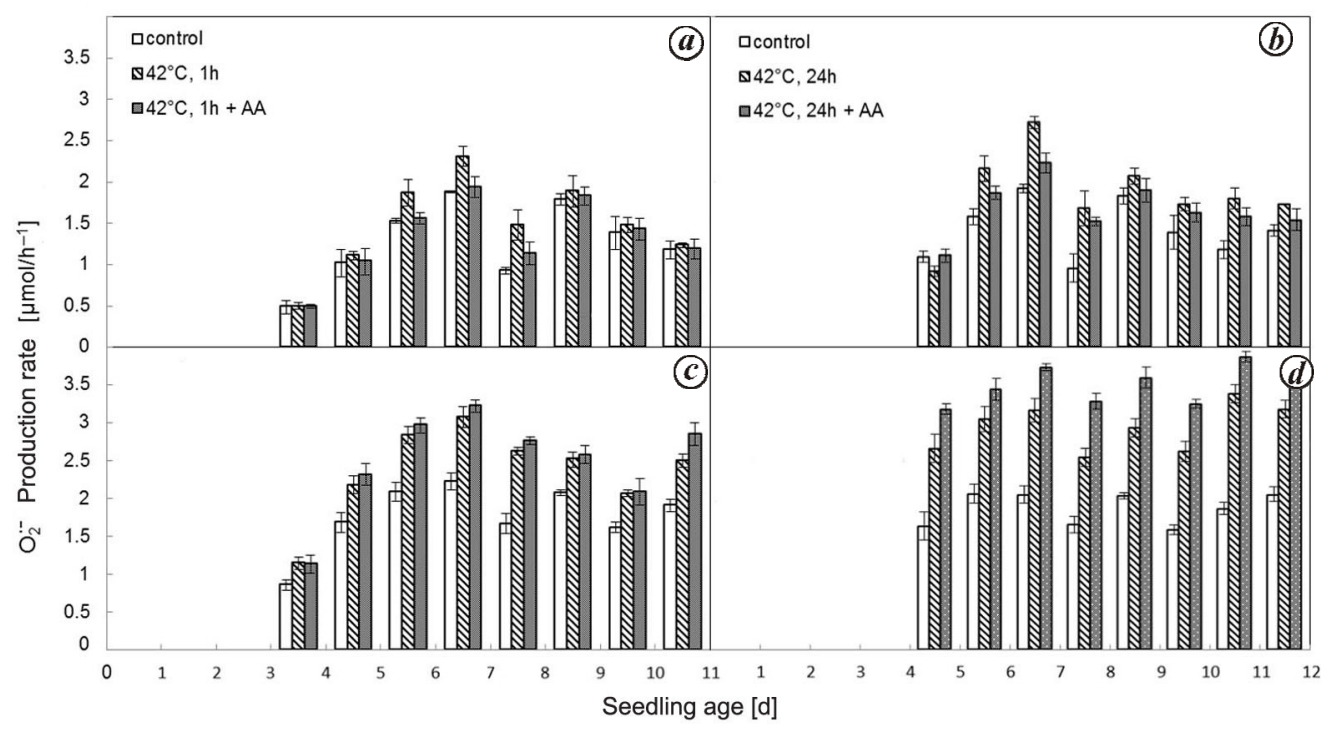

Figure 2. Effect of AA on $\mathrm{O}_{2}^{--}$production rate in the etiolated senescent organs grown under $(\boldsymbol{a})$ short-term and $(\boldsymbol{b})$ long-term exposure to high temperature, and under $(\boldsymbol{c})$ short-term and $(\boldsymbol{d})$ long-term exposure to high temperature under normal daylight conditions. Data are mean \pm SE $(n=3)$.

in the etiolated seedlings. In addition, PCD after HS $\left(50^{\circ} \mathrm{C}\right)$ was also accompanied by increased formation of ROS and mitochondrial inner membrane hyperpolarization ${ }^{15}$. Overall, the results of this study suggest that HT accelerates $\mathrm{O}_{2}^{--}$production in the senescent organs of wheat seedlings grown under normal daylight from 4 to 12 days of development that can be involved with PCD; but this warrants further studies.

The results also indicate that wheat seedlings exposed to AA treatment following short-term and long-term HT cause an increase in $\mathrm{O}_{2}^{\circ-}$ generation by $10 \%$ and $26 \%$ respectively, in the etiolated developing organs at different developmental stages compared with control seedlings (Figure $1 a$ and $b$ ). The increase in $\mathrm{O}_{2}^{--}$generation under the impact of AA and HT was not significant compared to stressful conditions without AA. During the development of etiolated wheat seedlings, the suppression level did not significantly alter under the impact of AA and HT. Thus, it is reasonable to assume that AA activated a nonphosphorylating AP which prevented an over-reduction state of cytochrome mETC and as a result reduced the level of ROS production in etiolated wheat seedlings. Maxwell and colleagues observed that AA led to a more efficient alternative respiration capacity ${ }^{9}$ and subsequently to a reduced mROS generation in tobacco cells ${ }^{24}$.

It is also noteworthy that the enhancement in the rate of $\mathrm{O}_{2}^{\circ-}$ generation in the etiolated senescent organs of wheat seedlings was not significant following the combined effect of AA and HT as under the stressful conditions without AA at all the developmental stages (Figure $2 a$ and $b$ ). We also found that this increase was more pronounced at the early stages of development. Our experimental data showed that AA reduced the rate of $\mathrm{O}_{2}^{--}$ generation on an average by $11 \%$ in the etiolated senes- cent organs following short-term and prolonged HT compared to stressful conditions without AA (Figure $2 a$ and $b)$. The above results demonstrate that AA alleviates the harmful effects of HT in the etiolated organs of wheat seedlings, indicating an induction of AP that reduces mROS production in all developmental stages and perhaps delays the senescence of whole coleoptiles. Previous studies demonstrated that the activation of AP reduced the production of hydrogen peroxide $\left(\mathrm{H}_{2} \mathrm{O}_{2}\right)$ and prevented or considerably delayed the senescence in Podospora anserine ${ }^{25}$. In addition, the activation of AP induced by AA effectively prevented ROS production in mETC in the etiolated winter wheat shoots under stressful conditions ${ }^{26}$. Thus, it can be assumed that the increased production of ROS accelerated the process of PCD in the senescent organs of wheat seedlings at the early and late stages of ontogenesis under exposure to HT, but an induction of the AP, provoked by AA, reduced substantially the $\mathrm{O}_{2}^{--}$producing rate in the etiolated senescent organs.

The present study also revealed that the rate of $\mathrm{O}_{2}^{\circ-}$ generation increased by $24 \%$ under the combined effect of AA and short-term HT, and by $42 \%$ under the combined effect of AA and prolonged HT in the developing organs of wheat seedlings grown under conditions of normal daylight compared to unstressed seedlings (Figure $1 c$ and $d$ ), suggesting the over-reduction of ETC around PSI in the chloroplasts ${ }^{27}$. It was previously demonstrated that accumulation of reducing equivalents (NADPH) by photochemical reaction in the stroma caused overreduction of the photosynthetic ETC and accelerated ROS generation in the chloroplasts ${ }^{28}$. A recent study reported that treatment of Nicotiana tabacum leaves with AA resulted in a substantial increase in the generation of $\mathrm{O}_{2}^{--}$ 


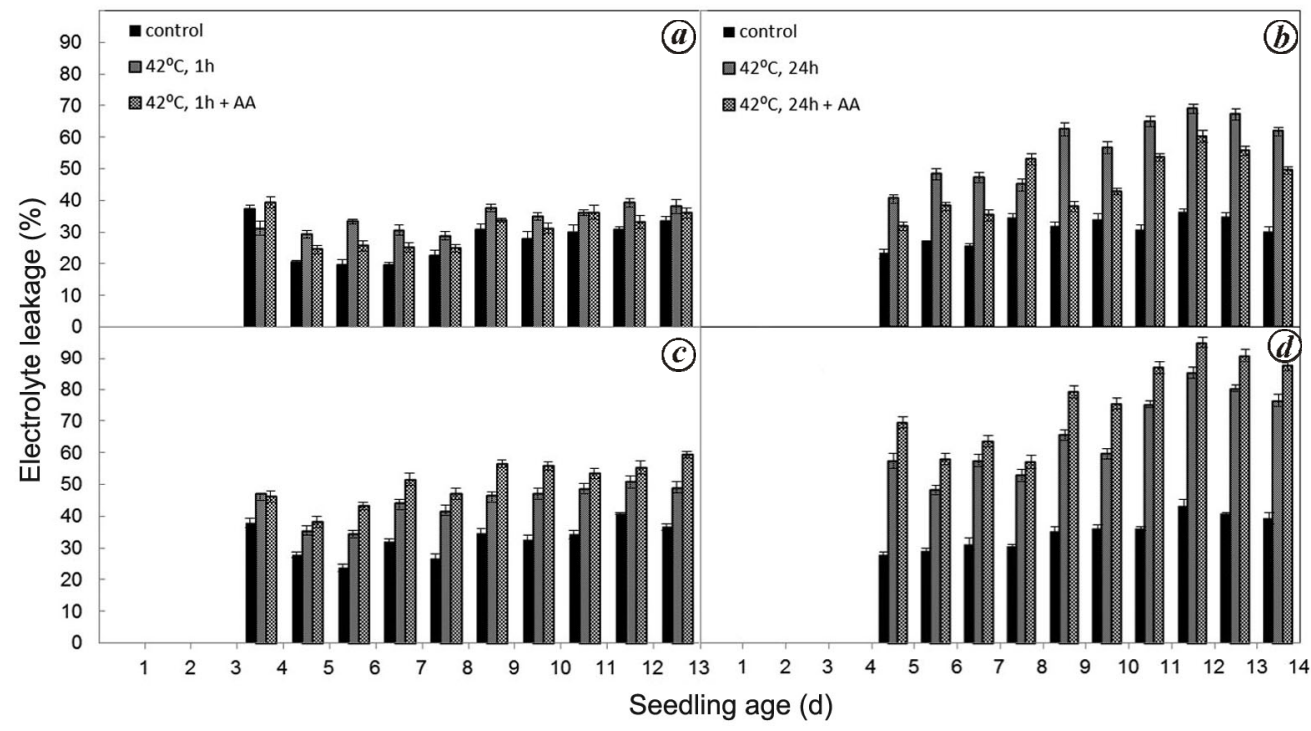

Figure 3. Effect of AA on electrolyte leakage in the etiolated developing organs grown under (a) short-term and (b) long-term exposure to high temperature, and under $(\boldsymbol{c})$ short-term and $(\boldsymbol{d})$ long-term exposure to high temperature under normal daylight conditions. Data are presented as mean $\pm \operatorname{SE}(n=3)$.

grown in a controlled-environment growth chamber with a $16 \mathrm{~h}$ photoperiod in comparison to control cells ${ }^{29}$.

Pilot studies revealed that the rate of $\mathrm{O}_{2}^{\circ-}$ generation increased on an average by $28 \%$ and $48 \%$ respectively, in the senescent organs of wheat seedlings subjected to AA following short-term and long-term HT and grown under normal daylight compared to control (Figure $2 c$ and $d$ ). This could be probably explained by the fact that AA inhibits cyclic electron transport around PS I in chloroplasts, leading to an increased rate of $\mathrm{O}_{2}^{\bullet-}$ production. Senescent organs of wheat seedlings were more sensitive to HT than the photosynthetically active leaves. A previous study reported that $\mathrm{H}_{2} \mathrm{O}_{2}$ content slightly increased after treatment with AA $(5 \mu \mathrm{M})$ in the senescent cell cultures (Arabidopsis thaliana) ${ }^{30}$. Overall, the enhanced rate of $\mathrm{O}_{2}^{--}$generation in the developing and senescent organs of wheat seedlings under AA treatment grown under conditions of normal daylight might be the result of inhibition of ferredoxin-dependent pathways of cyclic electron flow reactions around PSI in the chloroplasts.

\section{Changes in the permeability of cell membranes}

Membrane permeability is considered as an important indicator of the functional state of plants that varies during ontogenesis. The present study demonstrated that EL significantly increased by $24 \%$ in the etiolated developing organs following short-term HT regardless of the stages of development compared with control (Figure $3 a$ ), suggesting changes in the composition and structure of integral membrane proteins. It is known that considerable increase in electrolytes level under HT occurs due to major alterations of the tertiary and quaternary structures of membrane proteins ${ }^{31}$, and could be associated with the phase transition of polar lipids ${ }^{32}$. We estimate that the heat-induced ROS production led to an increase in membrane permeability in the developing organs of wheat seedlings. However, the increase in EL was more visible $(27 \%)$ in the developing organs under exposure to shortterm HT and grown under normal daylight conditions (Figure $3 c$ ), reflecting possibly strong thylakoid damages in photosynthesizing organs. Different photochemical reactions in the carbon flux in the space between the inner chloroplast membrane and grana, and those of the thylakoid membrane are regarding the primary sites of injury under exposure to $\mathrm{HT}^{33}$.

It should also be noted that the increase in EL in the etiolated developing organs was significantly greater when seedlings were exposed to prolonged HT compared with unstressed seedlings (Figure $3 b$ ). The present study revealed that EL increased by $45 \%$ and $47 \%$ respectively, following prolonged HT in the etiolated developing organs and those grown under normal light regime compared to control (Figure $3 b$ and $d$ ). EL in the etiolated developing organs and grown under normal daylight significantly increased at the late stages of development in comparison with the early stages of development (Figure $3 b$ and $d$ ). Taking into account that coleoptile functions for a relatively short period in cereals that protects leaves against an early stage of ontogenesis, it can be assumed that such an increase in membrane permeability in the developing organs at the late stages of development is associated with senescence of coleoptiles and an increase in oxidative stress that enhances the oxidative degradation of phospholipids, resulting in disruption of membrane permeability. These results agree with a previous study 


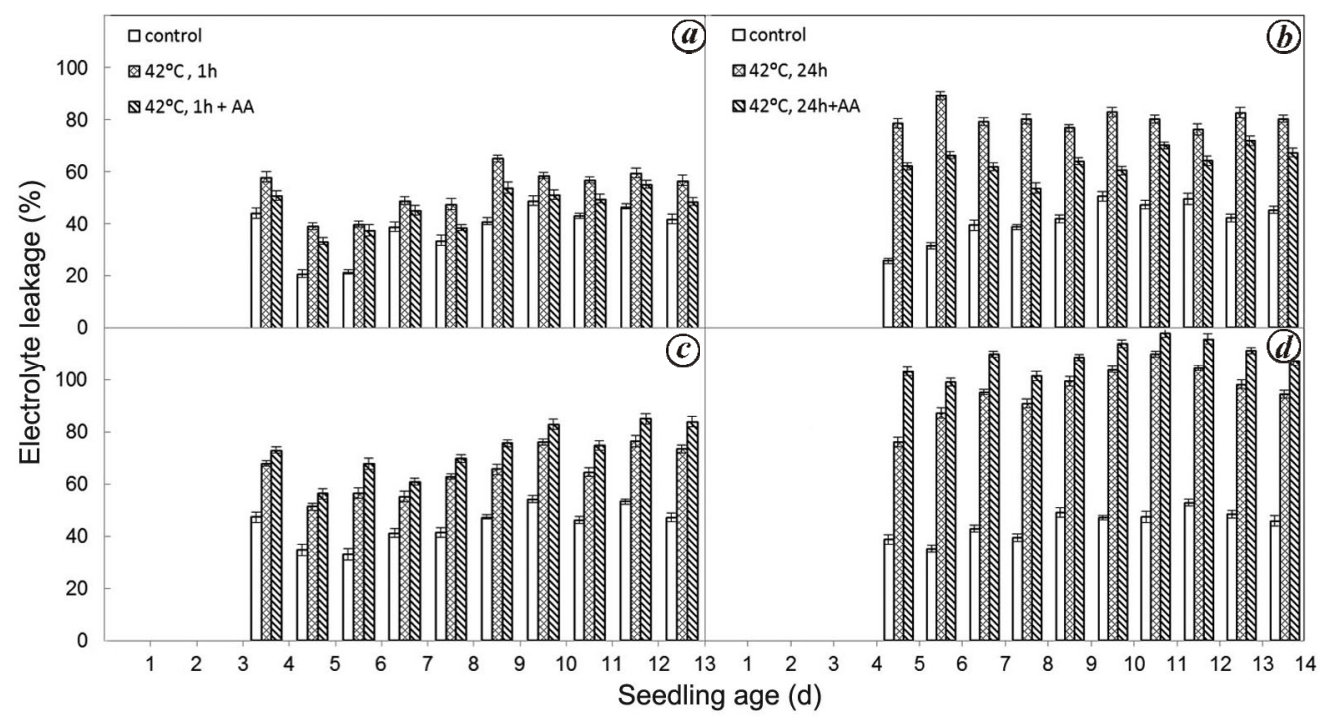

Figure 4. Effect of AA on electrolyte leakage in the etiolated senescent organs grown under (a) short-term and (b) long-term exposure to high temperature, and under $(\boldsymbol{c})$ short-term and $(\boldsymbol{d})$ long-term exposure to high temperature under normal daylight conditions. Data are presented as mean $\pm \operatorname{SE}(n=3)$.

on the elevated membrane permeability in leaves of $N$. tabacum subjected to long-term $\mathrm{HT}^{34}$.

The results of the present study demonstrate significant increase in EL by $29 \%$ in the etiolated senescent organs and by $31 \%$ in those grown under normal daylight under exposure to short-term HT (Figure $4 a$ and $c$ ). It should be emphasized that EL increased by $49 \%$ and $54 \%$ respectively, under prolonged HT in the etiolated senescent organs and those grown under normal daylight (Figure $4 b$ and $d$ ). It is likely that HT causes irreversible membrane damage in the senescent organs of wheat seedlings leading to a PCD and reduced plant viability. On the basis of these data, we conclude that the regulation system of membrane permeability and maintenance of homeostasis function is more effective in the developing organs of wheat seedlings. The results indicate that the increase in permeability of cellular membranes in the developing and senescent organs following HT occurs due to damage of membrane components. They also suggest that senescent organs of wheat seedlings are more susceptible to HT than the photosynthetically active leaves. Obviously, this could be the result of senescence - an increase in EL is a main symptom of senescence. It is also known that a large increase in EL under exposure to HT could be related to the occurrence of lipid peroxidation ${ }^{35}$. Previously, we reported an increase in the end-product of polyunsaturated fatty acid oxidation malondialdehyde in the senescent organs of wheat seedlings at the early stages of development in response to $\mathrm{HT}^{36}$. It is important to emphasize that an increase in EL under the combined effect of AA and HT is not significant compared to stressful conditions without AA in the etiolated developing organs. The present findings suggest that in wheat seedlings exposed to AA under exposure to short-term
HT, EL is lowered by $14 \%$ and $22 \%$ respectively, in the presence of AA under prolonged HT in the etiolated developing organs in comparison to those exposed to stressful conditions without AA (Figure $3 a$ and $b$ ). It could be assumed that the addition of AA causes a redistribution of the electron transport, leading to the activation of a nonphosphorylating AP, which in turn prevents the destruction of the membranes by lowering the EL and generating ROS in the etiolated organs of wheat seedlings following HT. Another study reported that EL was not altered by the addition of AA in etiolated cucumber cotyledons ${ }^{37}$. Thus, it can be assumed that the involvement of AP had some stabilizing effect on cellular membranes of etiolated wheat seedlings, reducing their permeability, maintaining cell homeostasis and playing a key role in maintaining cellular redox balance under unfavourable stress factors.

However, the results of this study indicate an increase $(14 \%)$ in EL in the developing organs in the presence of AA under exposure to short-term and long-term HT and grown under normal daylight (Figure $4 c$ and $d$ ) suggesting inhibition of the ferredoxin-dependent cyclic electron pathway $^{38}$. The results of the present study are in conformity with those of Strodtkötter et al. ${ }^{10}$, who reported that AA enhanced ROS ultimately resulted in amplified membrane leakage in leaf discs of $A$. thaliana cultivated in a

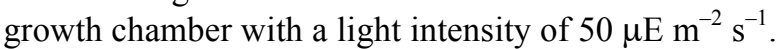

We also observed that the increase of EL was not as significant in the presence of AA under HT compared to stressful conditions without AA in the etiolated senescent organs of wheat seedlings (Figure $4 a$ and $b$ ). EL was found to decrease by $12 \%$ in the etiolated senescent organs following AA and short-term HT (Figure $4 a$ ). AA also caused a reduction $(20 \%)$ of EL in the etiolated senescent organs of wheat seedlings following prolonged 


\section{RESEARCH ARTICLES}

HT (Figure $4 b$ ). The present findings suppose that the induction of AP by AA increases the stability of cell membranes and might also contribute to the prevention of the ageing process in the senescent organs of wheat seedlings. However, experimental data show that AA increases EL on an average by $12 \%$ in the senescent organs grown under normal daylight and exposed to short-term and prolonged HT (Figure $4 c$ and $d$ ). Probably, the increased $\mathrm{O}_{2}^{\circ-}$ generation in the developing organs of wheat seedlings grown under normal daylight and conditions of HT and AA resulted in amplified membrane leakage compared to etiolated developing organs. Results of earlier studies demonstrated that AA caused membrane depolarization and a decrease of mitochondrial membrane potential in cells ${ }^{39}$. Overall these observations assumed that restriction of electron flow by AA enhanced the rate of generation of ROS in the chloroplasts, which in turn enhanced membrane permeability in wheat seedlings grown under normal daylight conditions.

\section{Conclusion}

In conclusion, the results of this study suggest that the rate of $\mathrm{O}_{2}^{--}$generation and membrane permeability increase following short-term and long-term HT in the developing and senescent organs of wheat seedlings presuming that HT contributes to the disruption of physiological processes in tissues and leads to oxidative stress. However, AA lowers the negative influence of HT in the etiolated organs of wheat seedlings. Furthermore, in contrast to the etiolated wheat seedlings, the intensity of oxidative stress is more pronounced in seedlings grown under normal daylight following the combined effect of AA and HT, suggesting inhibition of the ferredoxindependent pathways of cyclic electron flow reactions around PSI in chloroplasts of wheat seedlings.

1. Bita, C. E. and Gerats, T., Plant tolerance to high temperature in a changing environment: scientific fundamentals and production of heat stress-tolerant crops. Front. Plant Sci., 2013, 4, 1-18.

2. Wahid, A., Gelani, S., Ashraf, M. and Foolad, M. R., Heat tolerance in plants: an overview. Environ. Exp. Bot., 2007, 61, 199223.

3. Hasanuzzaman, M., Nahar, K., Alam, M., Roychowdhury, R. and Fujita, M., Physiological, biochemical, and molecular mechanisms of heat stress tolerance in plants. Int. J. Mol. Sci., 2013, 14, 9643 9684

4. Vanlerberghe, G. C., Alternative oxidase: a mitochondrial respiratory pathway to maintain metabolic and signalting homeostasis during abiotic and biotic stress in plants. Int. J. Mol. Sci., 2013, 14, 6805-6847.

5. Grabelnych, O. I. et al., Mitochondrial energy-dissipating systems (alternative oxidase, uncoupling proteins, and external $\mathrm{NADH}$ dehydrogenase) are involved in development of frost-resistance of winter wheat seedlings. Biochemistry (Mosc.), 2014, 79, 506-519.

6. Slane, B., Antimycin: the big bad chain blocker. Free Radic. Biol. Med., 2003, 77, 1-10.

7. Taira, Y., Okegawa, Y., Sugimoto, K., Abe, M., Miyoshi, H. and Shikanai, T., Antimycin A-like molecules inhibit cyclic electron transport around photosystem I in ruptured chloroplasts. FEBS Open Biol., 2013, 3, 406-410.

8. Vanlerberghe, G. C. and McIntosh, L., Coordinate regulation of cytochrome and alternative pathway respiration in tobacco. Plant Physiol., 1992, 100, 1846-1851.

9. Maxwell, D. P., Nickels, R. and McIntosh, L., Evidence of mitochondrial involvement in the transduction of signals required for the induction of genes associated with pathogen attack and senescence. Plant J., 2002, 29, 269-279.

10. Strodtkötter, I. et al., Induction of the AOX1D isoform of alternative oxidase in A. thaliana T-DNA insertion lines lacking isoform AOX1A is insufficient to optimize photosynthesis when treated with antimycin A. Mol. Plant, 2009, 2, 284-297.

11. Batjuka, A., Škute, N. and Petjukevičs, A., The influence of antimycin A on pigment composition and functional activity of photosynthetic apparatus in Triticum aestivum L. under high temperature. Photosynthetica, 2017, 55(2), 251-263.

12. Shorning, B. Y., Smirnova, E. G., Yaguzhinsky, L. S. and Vanyushin, B. F., Necessity of superoxide production for development of etiolated wheat seedlings. Biochemistry (Moscow), 2000, 65, 1357-1361.

13. Guo, Z., Ou, W., Lu, S. and Zhong, Q., Differential responses of antioxidative system to chilling and drought in four rice cultivars differing in sensitivity. Plant Physiol. Biochem., 2006, 44, 828836.

14. Grabel'nykh, O. I., Pobezhimova, T. P., Pavlovskaya, N. S., Koroleva, N. A., Borovik, O. A., Lyubushkina, I. V. and Voinikov, V. K., Antioxidant function of alternative oxidase in mitochondria of winter wheat during cold hardening. Biochemistry (Moscow) Suppl. Ser. A, 2014, 5, 249-257.

15. Lyubushkina, I. V., Fedyaeva, A. V., Pobezhimova, T. P., Stepanov, A. V. and Rikhvanov, E. G., Activation of cell death in the sugar cane suspension culture by the exposure to high temperature. J. Stress Physiol. Biochem., 2014, 10, 13-24.

16. Sharma, P., Jha, A. B., Dubey, R. S. and Pessarakli, M., Reactive oxygen species, oxidative damage, and antioxidative defense mechanism in plants under stressful conditions. J. Bot., 2012, 1, 26

17. Apel, K. and Hirt, H., Reactive oxygen species: metabolism, oxidative stress, and signal transduction. Annu. Rev. Plant Biol., 2004, 55, 373-399.

18. Gill, S. S. and Tuteja, N., Reactive oxygen species and antioxidant machinery in abiotic stress tolerance in crop plants. Plant Physiol. Biochem., 2010, 48, 909-930.

19. Fedyaeva, A. V., Stepanov, A. V., Lyubushkina, I. V., Pobezhimova, T. P. and Rikhvanov, E. G., Heat shock induces production of reactive oxygen species and increases inner mitochondrial membrane potential in winter wheat cells. Biochemistry (Moscow) Suppl. Ser. A, 2014, 79, 1202-1210.

20. Savicka, M. and Škute, N., Effects of high temperature on malondialdehyde content, superoxide production and growth changes in wheat seedlings (Triticum aestivum L.). Ekologija Liet. Moksl. Akad. (Spausd.), 2010, 56, 26-33.

21. Sairam, R. K. and Tyagi, A., Physiology and molecular biology of salinity stress tolerance in plants. Curr. Sci., 2004, 86, 407421.

22. Lee, R. H. and Chen, S. C. G., Programmed cell death during rice leaf senescence is nonapoptotic. New Phytol., 2002, 155, 25-32.

23. Roach, T. and Krieger-Liszkay, A., Regulation of photosynthetic electron transport and photoinhibition. Curr. Protein Pept. Sci., 2014, 15, 351-362.

24. Maxwell, D. P., Wang, Y. and McIntosh, L., The alternative oxidase lowers mitochondrial reactive oxygen production in plant cells. Proc. Natl. Acad. Sci. USA, 1999, 96, 8271-8276.

25. Dufour, E., Boulay, J., Rincheval, V. and Sainsard-Chanet, A., A causal link between respiration and senescence in Podospora anserina. Proc. Natl. Acad. Sci. USA, 2000, 97, 4138-4143. 
26. Grabelnych, O. I. et al., The participation of cyanide-resistant respiration in heat generation and antioxidative defense of cell in winter wheat shoots under cold influence. J. Stress Physiol. Biochem., 2011, 7, 446-456.

27. Geisler, D. A., Johansson, F. I., Svensson, Å. S. and Rasmusson, A. G., Antimycin A treatment decreases respiratory internal rotenone-insensitive NADH oxidation capacity in potato leaves. $B M C$ Plant Biol., 2004, 4, 1-10.

28. Yoshida, K., Terashima, I. and Noguchi, K., Up-regulation of mitochondrial alternative oxidase concomitant with chloroplast over-reduction by excess light. Plant Cell Physiol., 2007, 48, 606614.

29. Cvetkovska, M. and Vanlerberghe G. C., Alternative oxidase impacts the plant response to biotic stress by influencing the mitochondrial generation of reactive oxygen species. Plant Cell Environ., 2013, 36, 721-732.

30. Zentgraf, U., Zimmermann, P. and Smykowski A., Role of intracellular hydrogen peroxide as signalling molecule for plant senescence. In Senescence (ed. Nagata, T.), Intech Open, 2012, pp. $31-50$.

31. Al-Jebory, E. I., Changes in cellular membrane tolerance due to heat stress during Triticum sativum L. seeds germination. Mag. Al-Kufa Univ. Biol., 2013, 5, 1-9.

32. Bertin, P., Bouharmont, J. and Kinet, J. M., Somaclonal variation and improvement in chilling tolerance in rice: changes in chillinginduced electrolyte leakage. Plant Breed, 1996, 115, 268-272.

33. Wise, R. R., Olson, A. J., Schrader, S. M. and Sharkey, T. D., Electron transport is the functional limitation of photosynthesis in field-grown Pima cotton plants at high temperature. Plant Cell Environ., 2004, 27, 717-724.
34. Ivanov, S., Konstantinova, T., Parvanova, D., Todorova, D., Djilianov, D. and Alexieva, V., Effect of high temperature on the growth, free proline content and some antioxidants in tobacco plants. CR Acad. Bulg. Sci., 2001, 54, 71-74.

35. Ali, M. K., Azhar, A. and Galani, S., Response of rice (Oryza sativa L.) under elevated temperature at early growth stage: physiological markers. RJOAS, 2013, 8, 11-19.

36. Batjuka, A. and Škute, N., The effect of antimycin A on the intensity of oxidative stress, the level of lipid peroxidation and antioxidant enzyme activities in different organs of wheat (Triticum aestivum L.) seedlings subjected to high temperature. Arch. Biol. Sci., 2017, 69, 743-752.

37. Cho, K. Y., Kim, J. S. and Hong, K. S., Physiological action characteristics of $m$-substituted diphenylethers, TOPE and KC6361. Weed Technol., 1997, 42, 307-317.

38. Joët, T., Cournac, L., Horvath, E. M., Medgyesy, P. and Peltier, G., Increased sensitivity of photosynthesis to antimycin A induced by inactivation of the chloroplast $n d h B$ gene. Evidence for a participation of the NADH dehydrogenase complex to cyclic electron flow around photosystem I. Plant Physiol., 2001, 125, 1919-1929.

39. Kalbáčová, M., Vrbacký, M., Drahota, Z. and Mělková., Z., Comparison of the effect of mitochondrial inhibitors on mitochondrial membrane potential in two different cell lines using flow cytometry and spectrofluorometry. Cytometry A, 2003, 52, 110-116.

Received 29 January 2018; revised accepted 10 May 2019

doi: $10.18520 / \mathrm{cs} / \mathrm{v} 117 / \mathrm{i} 3 / 440-447$ 